\title{
Reducing the toxicity of lithium
}

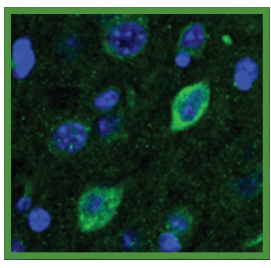

Lithium is the most effective treatment for bipolar disorder. However, its use is limited because of neurological side effects and a risk of overdose-induced toxicity. Many of the beneficial effects of lithium are thought to be mediated by its inhibition of glycogen synthase kinase-3 (GSK-3), but whether this is the mechanism underlying its negative effects has not been determined. To investigate this, Raquel Gómez-Sintes and José J. Lucas studied the effects of chronic administration of therapeutic doses of lithium to wildtype mice (2432-2445). This treatment regimen resulted in decreased GSK-3 activity in brain, gait abnormalities, and neuronal apoptosis in several regions of the brain. Further analysis showed increased nuclear localization of nuclear factor of activated T cells c3/4 (NFATc3/4) transcription factors and increased levels of Fas ligand in regions of the brain affected by neuronal apoptosis. Consistent with this, lithium-induced neuronal apoptosis and gait abnormalities were absent when nuclear translocation of NFAT was prevented by cyclosporin A administration and in Fasdeficient mice. The authors hope that their delineation of a molecular pathway by which lithium induces neuronal apoptosis and gait abnormalities in mice might provide new ideas for combination therapies that diminish the toxicities of lithium.

\section{Cancer in a PINCH(1)}

One of the main obstacles to complete elimination of a cancer is tumor cell resistance to ionizing radiation therapy and chemotherapy. One factor contributing to this is tumor cell adhesion to extracellular matrix mediated by macromolecular complexes known as focal adhesions. In this issue (2516-2527), Eke and colleagues find that particularly interesting new cysteine-histidine-rich 1 (PINCH1) functions downstream of focal adhesions to promote tumor cell radioresistance. Initial analysis indicated that PINCH1 expression was elevated in many different human tumors. The potential clinical importance of this was highlighted by the observation that mouse cells lacking PINCH1 showed enhanced sensitivity to ionizing radiation and a chemotherapeutic in vitro and to ionizing radiation in vivo. Further in vitro analysis uncovered the molecular mechanism of the prosurvival effect of PINCH1: it directly binds to protein phosphatase $1 \alpha(\mathrm{PP} 1 \alpha)$, which is an Akt1-regulating protein, and inhibits PP1 $\alpha$ activity, resulting in increased Akt1 phosphorylation and enhanced resistance to ionizing radiation. The authors therefore suggest that targeting molecules such as PINCH1 that function downstream of focal adhesions might provide new therapeutic approaches to overcoming tumor cell resistance to ionizing radiation therapy and chemotherapy.

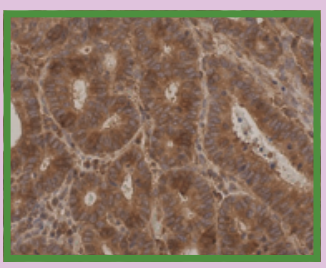

\section{Periostin promotes heart valve degeneration}

Valvular heart disease (VHD) is any disease process involving one or more of the heart valves. There are many causes of VHD, including atherosclerosis and rheumatic fever, but no therapies to prevent progressive valvular degeneration. Greater insight into the mechanisms underlying VHD pathogenesis is therefore needed. To address this issue, Hakuno and colleagues analyzed degenerated human cardiac valves from patients with either atherosclerotic or rheumatic VHD and found aberrant expression of periostin (2292-2306), a protein that plays critical roles in cardiac valve development. Specifically, periostin levels were markedly increased in infiltrated inflammatory cells and myofibroblasts in areas

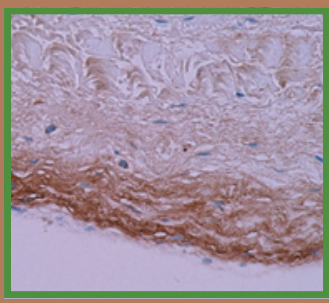
of angiogenesis. Consistent with these data having pathophysiologic relevance, the increased aortic valve defects observed in wild-type mice fed a high-fat diet were attenuated in mice lacking periostin. Further in vitro analysis indicated that periostin acts on endothelial cells to promote angiogenesis and on several cell types in the heart to promote secretion of MMPs, enzymes implicated in the progression of various degenerative diseases. These data provide new insight into the molecular mechanisms underlying VHD and suggest that periostin may be a therapeutic target to prevent progressive valvular degeneration in atherosclerotic and rheumatic VHD. 